\title{
Development of acute ischemic stroke and seizure in a patient with COVID-19 disease: A case report
}

\author{
Henil Upadhyay ${ }^{1}$, Charmy Parikh ${ }^{1}$, Dhruvrajsinh Chavda ${ }^{2}$, Roy Mali ${ }^{3}$ \\ From ${ }^{1}$ Intern, ${ }^{2}$ Resident, ${ }^{3}$ Professor, Department of Internal Medicine, Pramukhswami Medical College, Karamsad, Anand, Gujarat, India
}

\begin{abstract}
Acute ischemic stroke (AIS) is a life-threatening central nervous system (CNS) complication of coronavirus disease 2019 (COVID-19) infection. Here, we present the case of a 50-year-old COVID-19-positive male who developed AIS with hemorrhagic conversion during treatment in intensive care. He was managed with conservative treatment consisting of deep vein thrombosis prophylaxis and physiotherapy. We delayed oral aspirin and atorvastatin therapy due to hemorrhagic conversion. After 1 month of discharge, the patient presented with a seizure episode. He was observed for $24 \mathrm{~h}$ and discharged with prophylactic antiepileptic. Patients with COVID-19 may present with seizure episodes due to viral invasion into CNS, metabolic factors, and other systemic illnesses. Hence, seizures must be considered both as a presenting feature and long-term sequelae of COVID-19 infection. Physicians should take into consideration AIS as a possible complication of COVID-19 infection and should look out for clinical features suggestive of stroke for early diagnosis and improved clinical outcome.
\end{abstract}

Key words: COVID-19 disease, Hemorrhagic conversion, Seizure, Stroke

$\mathrm{T}$ The coronavirus disease 2019(COVID-19) is a viral infection primarily infecting the respiratory tract and is caused by the severe acute respiratory syndrome coronavirus 2 (SARS-CoV-2). It affects multiple organs including the nervous system [1]. Acute ischemic stroke (AIS) is a life-threatening central nervous system (CNS) complication of COVID-19 infection primarily mediated by inflammation, direct endothelial dysfunction, thrombin generation, and platelet activation [2]. According to a report the World Stroke Organization, the risk of ischemic stroke during COVID-19 infection is around 5\% [3].

This case report highlights the development of AIS postCOVID-19 infection complicated by hemorrhagic conversion and seizure. AIS can be a presenting feature of COVID-19 infection and should be kept as a differential diagnosis in patients with a strong clinical suspicion of COVID-19 infection.

\section{CASE REPORT}

A 50-year-old male presented with complaints of fever, breathlessness on exertion, and generalized weakness for a period of 4 days. His family and medical history were unremarkable.

On examination, his temperature was $99 \mathrm{~F}$, pulse rate was 96 beats/min, blood pressure was $120 / 80 \mathrm{mmHg}$, respiratory rate

\section{Access this article online}

Received - 20 February 2021

Initial Review - 09 March 2021

Accepted - 17 March 2021

DOI: $10.32677 /$ IJCR.2021.v07.i03.012 was $26 / \mathrm{min}$, and oxygen saturation was $88 \%$ on a non-rebreather mask with bilateral reduced air entry and basal lung crepitations.

The COVID-19 Reverse Transcription Polymerase Chain Reaction test was positive and the chest Computed Tomography (CT) scan showed multiple areas of ground-glass opacities, interstitial septal thickening, and consolidations involving bilateral lung parenchyma. The COVID-19 Reporting and Data System Score(CO-RADS) and the CT severity score were 5 and $15 / 25$ respectively. These clinical and radiological features were consistent with the changes of COVID- 19.

The patient was intubated in the intensive care unit for continued respiratory distress and hypoxia. The total leukocyte count was $13,000 / \mathrm{mm}^{3}$ (90\% neutrophils), C-reactive protein was $51 \mathrm{mg} / \mathrm{dL}$, prothrombin time was $79.90 \mathrm{~s}$, activated partial thromboplastin time was $40 \mathrm{~s}$, procalcitonin was $2.23 \mathrm{ng} / \mathrm{ml}$, and fibrinogen was $803 \mathrm{mg} / \mathrm{dl}$. He was prescribed remdesivir, tocilizumab, meropenem, colistin, and fluconazole.

On the $10^{\text {th }}$ day of hospitalization, he complained of the leftsided facial deviation, right upper and lower limb weakness. Physical examination showed impaired higher motor functions, right homonymous hemianopia, left gaze preference, right upper limb and lower limb flaccid weakness, right plantar extensor reflex, deep tendon reflexes $2+$ in all limbs, and no signs of meningeal irritation. The National Institutes of Health Stroke Scale (NISS) score was 21 signifying severe stroke.

Correspondence to: Dr. Henil Upadhyay, G-2/157, Ratanlal Park Soc, B/h Bapod Police Station, Ajwa-Waghodia Ring Road, Vadodara - 390 019, Gujarat, India. E-mail: upadhyayhenil@gmail.com

(C) 2021 Creative Commons Attribution-NonCommercial 4.0 International License (CC BY-NC-ND 4.0). 
CT brain suggested multiple acute infarcts with secondary hemorrhage foci at the left cerebellar hemisphere, left frontal and parietal lobe, and genu of the corpus callosum with mass effect and minimal midline shift with age-related changes of the cerebrocortical atrophy. Since ischemic stroke was associated with hemorrhagic conversion in this patient, we delayed oral aspirin and atorvastatin treatment for 2 weeks and monitored the patient for strict blood pressure control. He was also provided with deep vein thrombosis prophylaxis, physiotherapy to prevent muscle atrophy, and speech therapy.

After 2 weeks of stroke, atorvastatin (40 mg HS) and aspirin (75 mg OD) therapy was initiated which was continued on discharge from the hospital. He was kept on high-flow oxygen support due to COVID-19 viral pneumonia-associated hypoxia. After 3 weeks of hospitalization, the oxygen flow was gradually tapered off and the endotracheal tube was removed. Repeat highresolution $\mathrm{CT}$ of the thorax showed post-COVID lung fibrosis, and hence, the patient was given oral pirfenidone (200 mg TDS). Repeat CT brain after 2 weeks of stroke showed subacute infarct in the left superior cerebellar and left middle cerebral arterial territory with surrounding luxury perfusion (Fig. 1). Intracranial CT angiography showed thrombotic occlusion of few branches of M2 segment of the left middle cerebral artery (Fig. 2).

After 1 month of discharge, the patient presented with complaints of one episode of seizure, focal movement on the right upper and lower limb followed by tonic-clonic movements of all four limbs, uprolling of eyeballs, frothing from the mouth, and loss of consciousness for 10-15 min. Examination findings were right-sided residual upper motor neuron facial palsy and dysarthria, residual right-sided spasticity, power $1 / 5$ in the right upper limb and $4 / 5$ in the right lower limb, brisk deep tendon reflexes on the right side, bilateral plantar extensor reflex, leftsided dysmetria, and dysdiadochokinesia. Electroencephalogram showed abnormal awake record with intermittent generalized epileptiform discharge. CT brain showed early chronic infarct in the left superior cerebellar and the left middle cerebral arterial territory. He was given intravenous levetiracetam on presentation in emergency care and was observed for $24 \mathrm{~h}$. He was discharged with prophylactic antiepileptic as he was vitally stable and there were no episodes of seizures.

\section{DISCUSSION}

The SARS-CoV-2 viral pandemic began as a cluster of pneumonia cases of unknown origin in Wuhan, China, and has gradually spread across the globe. Human transmission occurs through aerosol spread or direct contact with a patient harboring the virus. Although interstitial pneumonia with ground-glass opacities is the predominant manifestations, there is increasing evidence that the virus invades all components of the body including the central nervous system [4].

AIS is one of the manifestations in CNS involvement of COVID-19 infection. The incidence of AIS in COVID-19 patients ranges from $0.9 \%$ to $2.7 \%$. The proposed mechanisms for stroke

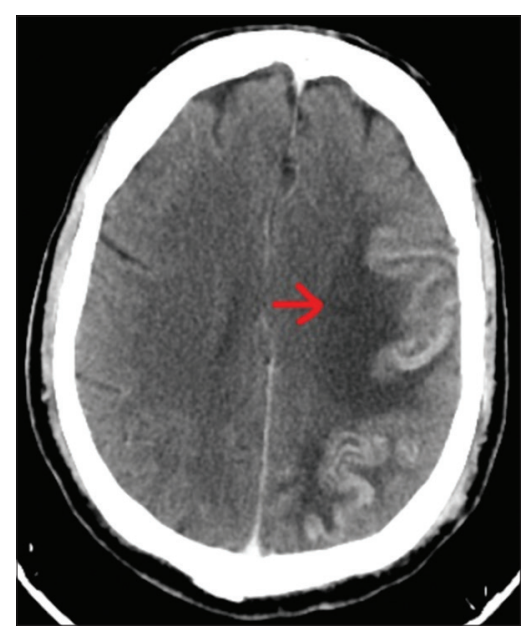

Figure 1: CT scan of brain showing subacute infarct in the left middle cerebral artery territory with luxury perfusion

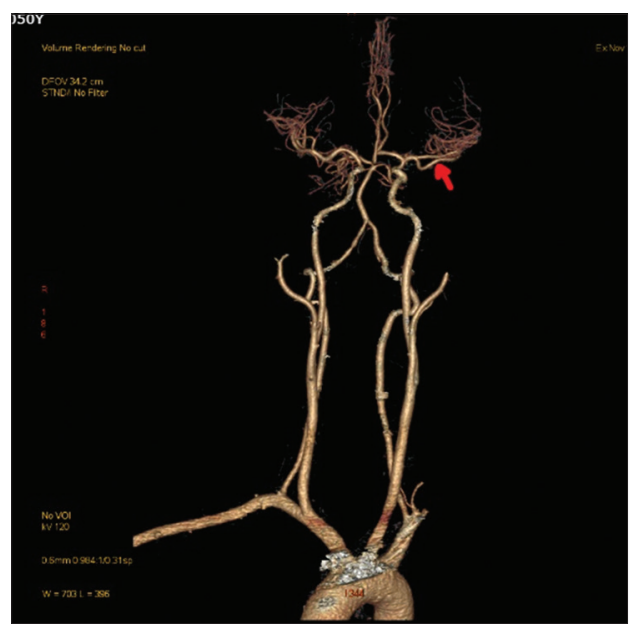

Figure 2: Cervical CT angiography showing thrombotic occlusion of few branches of M2 segment of the left middle cerebral artery

include hypoxemia and inflammation. It has been hypothesized that COVID-19 infection-induced hypoxemia and release of inflammatory cytokines cause cell ischemia and tissue damage including apoptosis [5]. The mean duration of AIS from the onset of COVID-19 symptoms as reported in the literature is $10 \pm 8$ days which suggests delayed presentation and is similar to the timeline in our case. A study from New York showed that COVID-19 did not increase the risk of stroke on presentation although no definite conclusions could be drawn from the study [5].

Respiratory viral infections such as the influenza are known to increase the risk for ischemic stroke by mechanisms of inflammation, pro-thrombotic coagulopathy, and endothelial injury. Similarly, COVID-19 also causes cytokine storm and inflammation as evident by elevated levels of D-dimer and anti-phospholipid antibodies [6]. The cytokine storm here is mediated through binding of SARSCoV-2 to angiotensin-converting enzyme 2 receptor [7]. Secondary hemorrhagic complications are more common in COVID-19related ischemic strokes as there is pre-existing endothelial damage and consumption coagulopathy [3].

Patients with COVID-19 may present with multiple seizure episodes. Contributing factors for seizure include viral invasion 
into CNS, metabolic factors, and other systemic illnesses [8]. Infarct-related changes in the brain can lead to seizures in the long term. Hence, seizures must be considered both as a presenting feature and long-term sequelae of COVID-19 infection.

\section{CONCLUSION}

AIS should be taken into consideration by the physicians as a possible complication of COVID-19 infection and should look out for clinical features suggestive of stroke for early diagnosis and improved clinical outcome. Laboratory parameters such as platelet counts and D-dimer levels can help in charting the progress of the disease during the course of illness. It is also advisable to delay thrombolytic therapy and endovascular treatment in cases of stroke with a hemorrhagic conversion.

\section{REFERENCES}

1. Gunasekaran K, Amoah K, Rajasurya V, Buscher MG. Stroke in a young COVID-19 patient. QJM 2020;113:573-4.

2. Ntaios G, Michel P, Georgiopoulos G, Guo Y, Li W, Xiong J, et al. Characteristics and outcomes in patients with COVID-19 and acute ischemic stroke: The global COVID-19 stroke registry. Stroke 2020;51:e254-8.

3. Spence JD, De Freitas GR, Pettigrew LC, Ay H, Liebeskind DS, Kase CS, et al. Mechanisms of stroke in COVID-19. Cerebrovasc Dis 2020;49:451-8.

4. Tan YK, Goh C, Leow AS, Tambyah PA, Ang A, Yap ES, et al. COVID19 and ischemic stroke: A systematic review and meta-summary of the literature. J Thromb Thrombolysis 2020;50:587-95.

5. Bekelis K, Missios S, Ahmad J, Labropoulos N, Schirmer CM, Calnan DR, et al. Ischemic stroke occurs less frequently in patients with COVID-19: A multicenter cross-sectional study. Stroke 2020;51:3570-6.

6. Merkler AE, Parikh NS, Mir S, Gupta A, Kamel H, Lin E, et al. Risk of ischemic stroke in patients with coronavirus disease 2019 (COVID-19) vs patients with influenza. JAMA Neurol 2020;77:1366-72.

7. Belani P, Schefflein J, Kihira S, Rigney B, Delman BN, Mahmoudi K, et al. COVID-19 is an independent risk factor for acute ischemic stroke. Am J Neuroradiol 2020;41:1361-4

8. Anand P, Al-Faraj A, Sader E, Dashkoff J, Abdennadher M, Murugesan R, et al. Seizure as the presenting symptom of COVID-19: A retrospective case series. Epilepsy Behav 2020;112:107335.

Funding: None; Conflicts of Interest: None Stated.

How to cite this article: Upadhyay $\mathrm{H}$, Parikh C, Chavda D, Mali R. Development of acute ischemic stroke and seizure in a patient with COVID-19 disease: A case report. Indian J Case Reports. 2021;7(3):111-113. 